\title{
Managing Gout in Women: Current Perspectives
}

\author{
Aakash V Patel', Angelo L Gaffo $\mathbb{D}^{2,3}$ \\ 'Tinsley Harrison Internal Medicine Residency Program, University of Alabama at Birmingham, Birmingham, AL, USA; ${ }^{2}$ Division of Rheumatology and \\ Clinical Immunology, University of Alabama at Birmingham, Birmingham, AL, USA; ${ }^{3}$ Birmingham VA Medical Center, Birmingham, AL, USA \\ Correspondence: Angelo L Gaffo, Division of Rheumatology and Clinical Immunology, University of Alabama at Birmingham, 1825 Shelby Biomedical \\ Building, Birmingham, AL, 35294, USA, Tel +I 205-933-8I0I, Fax + I 205-996-6788, Email agaffo@uabmc.edu
}

\begin{abstract}
Gout is a common inflammatory arthritis that tends to affect significantly more men than women. However, female gout patients are more likely to have comorbidities such as hypertension, diabetes mellitus, and renal dysfunction. Furthermore, they experience a greater disease burden due to gout than males. While nonbiological causes may possibly contribute to this sex discrepancy in burden, this raises questions regarding whether current gout pharmacotherapies are as efficacious in females as they are in males. In this review, we examine how the clinical profile of female gout patients differs from male patients; we then survey the literature for data on outcomes for female gout patients treated with urate-lowering therapies for chronic management of gout as well as commonly used agents for acute flares. We also discuss considerations for managing gout in women during pregnancy and lactation.
\end{abstract}

Keywords: gout, women, treatment, flare, prophylaxis, pregnancy

\section{Introduction}

Gout is the most common inflammatory arthritis with an overall prevalence of $1-4 \%$ worldwide; ${ }^{1}$ it is caused by monosodium urate crystal deposition in synovial fluid and other tissues as a result of elevated serum urate levels. ${ }^{2}$ The subsequent inflammatory response to the monosodium urate crystals leads to synovitis and severe arthralgias, and complications can include tophus deposition and chronic arthritis. Serum urate-lowering therapies serve as the mainstay of treatment. ${ }^{3,4}$

In some populations, gout has been shown to affect up to ten times more males than females. ${ }^{1,5}$ Gout in women typically occurs in post-menopause, with an average age of diagnosis at 65-71 years vs 61-62 years in males, ${ }^{6,7}$ consistent with the known serum urate lowering properties of both estrogen and progesterone. ${ }^{8}$ Gout in pregnancy is also markedly uncommon; there were no cases of gout in a study of 190 pregnant females, of whom $46 \%$ had the ABCG2 allele, which is known to be associated with hyperuricemia. ${ }^{9}$ However, the incidence of gout in elderly males and females approximate each other more closely. For example, in one study, the male-to-female ratio of the incidence of gout in individuals over the age of 70 was noted to be just 2.3. ${ }^{10}$

Gout has a strong genetic basis that drives disease activity. In particular, there are polymorphisms in two genes, PDZK1 and $A B C G 2$, that are associated with hyperuricemia, although the functional manifestations of these polymorphisms differ between males and females. ${ }^{11}$ Specifically, the polymorphisms in $A B C G 2$ and PDZK1 confer a disproportionately increased rate of hyperuricemia when present in males compared to females.

Female gout patients are generally afflicted by multiple other comorbidities. Although there is a higher prevalence of the metabolic syndrome in both male and female gout patients, ${ }^{12}$ affected females are more likely than males to have concomitant hypertension ( $77-78 \%$ vs $56-57 \%$ ), diabetes mellitus ( $28-39 \%$ vs $17 \%$ ), renal dysfunction (24-64\% vs 13-31\%), and increased BMI (average BMI 33.5 vs 31.9). ${ }^{6,7}$ The increased presence of these and other comorbidities, including stroke, heart failure, coronary heart disease, and sleep apnea, as determined by higher odds ratios, have been recently corroborated in a large United Kingdom Biobank cohort. ${ }^{13}$ 
Most importantly, while serum urate levels and the rates of podagra, tophi, monoarthritis, polyarthritis, and flares have been shown to be comparable between males and females with gout, ${ }^{7}$ women report greater disability, when assessed with Health Assessment Questionnaire scores, as a sequelae of their gout ${ }^{7}$ a significantly greater proportion of affected females than males require treatment with analgesics, including opioid-type pain relievers, during flares. This discrepancy in burden experienced by female gout patients could possibly be due to how they are less likely to be prescribed allopurinol and receive a definitive diagnosis of gout through synovial fluid crystal analysis compared to their male counterparts; they also have higher rates of concurrent thiazide diuretic use. ${ }^{7}$ Nonetheless, the discrepancy in gout burden among females raises the obvious question regarding whether current gout pharmacotherapies are as efficacious in females as they are in males. Herein, we review the literature for outcomes data for gout pharmacotherapies in women.

\section{Treatment of Gout in Women}

Given the central role of hyperuricemia in the pathophysiology of gout, urate lowering therapies, including xanthine oxidase inhibitors, uricosuric agents, and uricases, are employed with the goal of preventing flares, which in turn are most commonly managed with nonsteroidal anti-inflammatory drugs (NSAIDs), colchicine, glucocorticoids, or anti-IL$1 \beta$ agents. ${ }^{14}$ The evidence for the above therapies in treating female gout will be reviewed here.

\section{Urate Lowering Therapy for Chronic Gout}

In gout, hyperuricemia is generally secondary to decreased renal or gastrointestinal clearance, rather than primary overproduction, of uric acid. The European League Against Rheumatism (EULAR) and American College of Rheumatology (ACR) both support a urate-lowering approach for patients with gout who have had greater than one flare in a year. ${ }^{4,15}$ The ACR recommends treating to a goal serum urate level of less than $6.0 \mathrm{mg} / \mathrm{dl}$. No professional guidelines or other evidence suggest any benefit in treating female gout patients to a different goal serum urate level.

\section{Xanthine Oxidase Inhibitors}

By definition, xanthine oxidase inhibitors prevent production of urate, which leads to a reduction in serum urate levels; as such, they are capable of facilitating crystal dissolution while also preventing further crystal deposition, proportional to the amount of urate lowering achieved. ${ }^{16}$ Allopurinol, a competitive inhibitor, and febuxostat, a noncompetitive inhibitor, are the two most commonly used xanthine oxidase inhibitors and serve as first-line therapies for the treatment of gout in all populations. ${ }^{4}$ Due to uncertainty regarding associated increased cardiac risks with febuxostat use and cost considerations, the ACR currently recommends initiating therapy first with allopurinol in all populations and reserving febuxostat for patients who experience adverse events, such as allopurinol hypersensitivity syndrome, or are recalcitrant to allopurinol. ${ }^{4}$ A retrospective analysis compiled data from three different Phase III clinical trials \{Febuxostat versus Allopurinol Controlled Trial (FACT), ${ }^{17}$ Allopurinol- and Placebo-Controlled, Efficacy Study of Febuxostat (APEX), ${ }^{18}$ and CONFIRMS ${ }^{19}$ \}, which collectively contained 226 female gout patients with serum urate levels greater than or equal to $8.0 \mathrm{mg} / \mathrm{dl}$, to examine the efficacy of varying doses of allopurinol and febuxostat for the treatment of female gout patients. ${ }^{20}$ Study participants in the three trials had been randomized to receive either allopurinol (at doses of $100 \mathrm{mg}$, $200 \mathrm{mg}$, or $300 \mathrm{mg}$ daily based on renal function), febuxostat $(40 \mathrm{mg}, 80 \mathrm{mg}, 120 \mathrm{mg}$, or $240 \mathrm{mg}$ daily), or placebo. Participants were treated for a duration of 24 to 52 weeks following randomization. The primary endpoint utilized by the retrospective study was the proportion of patients with a serum urate level less than $6.0 \mathrm{mg} / \mathrm{dl}$ at the final visit. The proportion of females who achieved this was statistically greater among those who were treated with febuxostat $80 \mathrm{mg}$ $(85.1 \%)$ or $120 \mathrm{mg}(81.0 \%)$, compared to febuxostat $40 \mathrm{mg}(54.3 \%)$ or any dose of allopurinol $(45.9 \%)$, even when the female patients were stratified by renal function (Figure 1). In regard to safety profile, three out of 139 female patients on febuxostat, and three out of 76 patients on allopurinol, experienced a cardiac serious adverse event.

To our knowledge, the above represents the only publicly available data that can be used specifically to evaluate treatment outcomes in female gout patients with xanthine oxidase inhibitors. The retrospective study performed post-hoc analyses on the three individual phase III clinical trials, none of which were inherently powered to evaluate response or adverse effects to treatment with allopurinol or febuxostat among females. In addition, these studies did not utilize doses of allopurinol greater than $300 \mathrm{mg}$ daily. Patients commonly need doses greater than $300 \mathrm{mg}$ per day, up to the maximum 


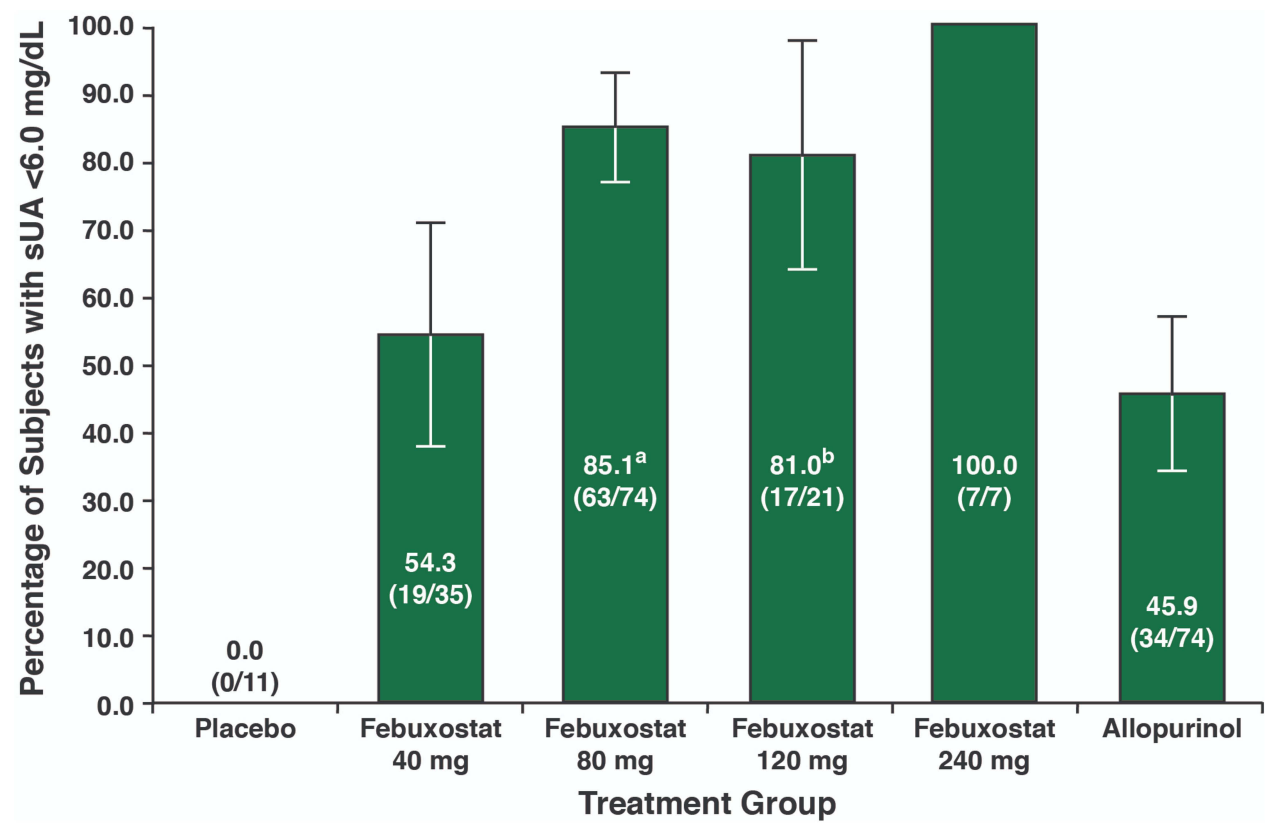

Figure I Percentage of female gout patients with a serum urate level less than $6.0 \mathrm{mg} / \mathrm{dl}$ after treatment with febuxostat and allopurinol. Two subjects with a creatinine clearance of less than $60 \mathrm{~mL} /$ minute received allopurinol $100 \mathrm{mg}$ and 32 received allopurinol $200 \mathrm{mg}$, while the remaining subjects received allopurinol $300 \mathrm{mg}$. The $95 \%$ confidence intervals are indicated by the error bars. Reproduced from Chohan S, Becker MA, MacDonald PA, Chefo S, Jackson RL. Women with gout: efficacy and safety of urate-lowering with febuxostat and allopurinol. Arthritis Care Res (Hoboken). 2012;64(2):256-26I. Copyright @) 2012 by the American College of Rheumatology. ${ }^{20}$ aWhen using Fisher's exact test to compare to the allopurinol group, $P<0.00 \mathrm{I}$. 'When using Fisher's exact test to compare to the allopurinol group, $P=0.006$.

dose of $800 \mathrm{mg}$ per day, to achieve goal serum urate concentrations, ${ }^{4}$ and this could also be true for female gout patients in particular.

Of note, a separate study investigated the pharmacokinetics, pharmacodynamics, and safety of febuxostat $80 \mathrm{mg}$ once daily between healthy males and females. ${ }^{21}$ There were no differences in any of the parameters. However, the major limitation of this analysis was that all study participants were healthy; as such, conclusions cannot be extrapolated to the female gout population.

From a safety perspective, the occurrence of adverse events in female febuxostat and allopurinol users was comparable to those among participants in the overall FACT, CONFIRMS, and APEX trial populations. Additionally, a separate study specifically comparing the rate of adverse events between male and female gout patients using febuxostat did not reveal any significant differences. ${ }^{22}$ In terms of cardiovascular safety, various studies, none of which are stratified by sex, have demonstrated mixed findings with regard to whether febuxostat carries greater cardiovascular risks compared to allopurinol. ${ }^{23-25}$ Fortunately, data from the recent Febuxostat versus Allopurinol Streamlined Trial (FAST) has been more reassuring about the cardiovascular safety of febuxostat. ${ }^{26}$ This was a randomized, blinded-endpoint noninferiority trial containing 6128 male and female gout patients, with at least one additional cardiovascular risk factor, from Denmark, United Kingdom, and Sweden who received either allopurinol or febuxostat. The primary endpoint was hospitalization for non-fatal myocardial infarction, biomarker positive acute coronary syndrome, nonfatal stroke, and cardiovascular death. Findings demonstrated that febuxostat was noninferior to allopurinol, with 1.72 compared to 2.05 events per 100 patient years among febuxostat and allopurinol users, respectively. As described above, female gout patients have a higher rate of comorbid cardiovascular risk factors compared to their male counterparts and thus, additional studies specifically assessing the cardiovascular safety of xanthine oxidase inhibitors in female gout patients will be crucial in guiding further clinical decision-making for this demographic. Ultimately, shared decision-making between female gout patients and physicians will be crucial when initiating urate lowering therapy with a xanthine oxidase inhibitor, particularly for those who have cardiovascular comorbidities. 


\section{Uricosuric Agents}

Probenecid and lesinurad are two agents that both inhibit the renal transporter URAT-1, which leads to urate lowering via uricosuria. Lesinurad is no longer commercially available in the United States, leaving probenecid as the only readily available pharmacologic agent in this class. Probenecid can be used alone or in combination with a xanthine oxidase inhibitor, depending on the severity of disease. It should be noted that renal disease precludes the use of uricosuric agents. A retrospective observational study assessing the efficacy of probenecid included 13 females and 17 males, all of whom were treated with probenecid monotherapy. ${ }^{27}$ Collectively, ten out of 30 patients achieved target serum urate levels, the primary endpoint of the study. It is unclear how many of the non-responders were males compared to females, as the data were not stratified by sex. Probenecid is not as widely used as xanthine oxidase inhibitors, and as expected, there is only minimal data on the efficacy in females.

\section{Uricases}

Rasburicase and pegloticase are both uricases, which degrade uric acid. There are multiple individual case reports documenting the marked urate lowering efficacy of rasburicase in female patients who were intolerant or unresponsive to allopurinol. ${ }^{28-30}$ However, rasburicase's immunogenicity prevents it from serving as a sustainable treatment option, as host antibodies directed against the agent increase with usage. Pegloticase is less immunogenic due to the polyethylene glycol component, although patients can still develop antibodies to polyethylene glycol, leading to infusion reactions. These can be mitigated with concomitant immunosuppressive therapy. Two replicate, randomized, placebo-controlled six-month trials and an open-label treatment extension study that led to the approval of pegloticase contained a total of 38 females and 172 males, but the results were not stratified by sex; ${ }^{31,32}$ as such, conclusions regarding the efficacy of pegloticase in females involved in this study cannot be made. However, a descriptive study consisting of three females with refractory gout assessed baseline and post-pegloticase therapy serum urate levels and tophus volumes, as measured by dual-energy CT scans. ${ }^{33}$ Two of the female participants were considered responders, defined by those who maintained a serum urate level less than $6.0 \mathrm{mg} / \mathrm{dl}$ for at least $80 \%$ of the study duration; these two patients further exhibited 96.96$100 \%$ of tophus volume reduction. The third female patient was considered a partial-responder with a $73.10 \%$ reduction in tophus volume. Owing to the relative recency of pegloticase's approval for gout, there is minimal data on the effectiveness of pegloticase in females in particular.

\section{Management of Acute Flares}

The 2020 ACR guidelines recommend treating acute gout flares with NSAIDs, colchicine, and glucocorticoids (systemic or intraarticular), in addition to supportive care with topical ice. ${ }^{4}$ For cases refractory to the above therapies, treatment with anti-IL1B therapy, namely anakinra, is indicated. ${ }^{4}$ Similar to the chronic therapies above, there is only limited female-specific data for the acute therapies. Published gout medication usage data demonstrate that comparable proportions of males and females utilize colchicine, oral steroids, and NSAIDs.

Indomethacin is the preferred NSAID for acute gout management. ${ }^{34} \mathrm{~A}$ pooled-subgroup analysis in which patients experiencing an acute gout flare were randomized to receive indomethacin $50 \mathrm{mg}$ three times a day compared to etoricoxib 120mg daily (now discontinued in the United States) contains the only known female-specific data for NSAID use in acute gout. ${ }^{35}$ In total, 147 males and 14 females received indomethacin 50mg three times a day, and the subgroup analysis did not reveal any difference in primary endpoint efficacy, defined by self-reported pain ratings, between male and female patients. ${ }^{35}$ Importantly, there are cardiovascular risks associated with NSAID use; upregulation of the COX1 pathway following the use of selective and even non-selective cyclooxygenase inhibitors, such as indomethacin, leads to vasoconstriction and platelet aggregation, ultimately facilitating intravascular thrombosis. Therefore, among female gout patients who are vulnerable to cardiovascular disease due to their higher rates of predisposing comorbidities, NSAID use should be employed cautiously even if only used during acute flares. ${ }^{7}$ Similarly, utilization in patients with renal disease, which is more prevalent in female gout patients, should be avoided. ${ }^{7}$

Colchicine inhibits tubulin aggregation, thereby downregulating inflammatory pathways. A multicenter, randomized, double-blind, placebo-controlled, parallel-group, dose-comparison study assessed the efficacy of low-dose vs high-dose 
colchicine, compared to placebo, for the treatment of 185 patients, including nine females, experiencing a gout flare. ${ }^{36}$ The primary endpoint was defined as a greater than or equal to $50 \%$ reduction of pain without the use of rescue medications. Approximately $38 \%$ of patients in the low-dose colchicine group, compared to $33 \%$ in the high-dose and $16 \%$ in the placebo group, were considered responders. Although there is no sex-specific stratified data available, a computation of the odds ratio for confounding bias suggested that demographic parameters, such as age, sex, and race, were not confounders.

Colchicine may also serve a secondary purpose in female gout patients. A meta-analysis of five studies containing 11,816 coronary artery disease patients who were randomly assigned to a low-dose colchicine or placebo treatment group demonstrated that low-dose colchicine led to a reduction in major adverse cardiac events, specifically myocardial infarction, stroke, and the need for coronary revascularization. ${ }^{37}$ In addition, a randomized-controlled trial assessing the efficacy of colchicine in chronic coronary disease did not reveal difference in efficacy between males and females. ${ }^{38}$ Therefore, female gout patients, who have higher rates of predisposing comorbidities such as hypertension and diabetes mellitus than male patients, may further benefit from colchicine therapy, although studies regarding the cardioprotective properties of colchicine remain ongoing. ${ }^{7}$

Intraarticular and systemic steroids serve as additional treatment options for patients with acute gout flares, particularly those with renal disease. In one prospective trial, 12 patients with acute gout flares were treated with oral prednisone or methylprednisolone. ${ }^{39}$ Four of these were female patients treated with an oral prednisone taper starting anywhere between 20 and $40 \mathrm{mg}$ daily, with complete resolution of symptoms occurring between four and nine days. One additional female patient was treated with an initial dose of methylprednisolone 50mg and had complete resolution of symptoms after six days. Unfortunately, to our knowledge, there is no available data on the efficacy of intraarticular steroids for treatment of acute gout flares specifically in female patients.

Anakinra, the newest therapeutic for acute gout and an anti-IL1 $\beta$ agent, serves as a second-line treatment option for those unresponsive to the above therapies. In the initial pilot, open-label study, ten patients, including two females, were treated with anakinra $100 \mathrm{mg}$ daily for three days for acute gout after failing the above therapies. ${ }^{40}$ The two female patients reported a $70-80 \%$ reduction in pain after treatment with anakinra. Furthermore, a retrospective chart review of ten patients treated with anakinra for acute gout contained two females, both of whom had a "good" response, defined as complete or near complete resolution of joint symptoms. ${ }^{41}$ A double-blind, placebo-controlled, active-comparator, noninferiority trial consisted of 88 patients, including five females, but did not stratify findings by sex. ${ }^{42}$ Anakinra has recently been increasingly utilized in hospitalized patients with numerous comorbidities that preclude the use of the above therapies. A retrospective chart review of 13 ICU patients being treated with anakinra for acute gout included one female patient, who reportedly had "a significant response". 43 The largest known observational study assessing the efficacy of anakinra for the treatment of acute gout in hospitalized patients did not stratify by sex. ${ }^{44}$

\section{Special Considerations: Pregnancy and Lactation}

Gout in pre-menopausal women is a rare occurrence and often manifests in patients with inherited conditions such as familial juvenile hyperuricemic nephropathy. ${ }^{45,46}$ Underlying kidney disease and gestational diabetes are other predisposing factors for the onset of gout in pre-menopause. ${ }^{47}$ Pregnant and breastfeeding women with acute gout flares should generally be treated with glucocorticoids; these may be used cautiously or contraindicated in those with gestational hypertension or gestational diabetes, depending on severity. NSAIDs can be utilized for the treatment of acute flares during lactation and the first two trimesters of pregnancy but are strictly contraindicated in the third trimester as they can cause premature closure of the ductus arteriosus. On the contrary, colchicine is generally avoided altogether during both pregnancy and lactation due to reports of colchicine-induced chromosomal damage and secretion into breastmilk. ${ }^{48,49} \mathrm{In}$ regard to chronic management, women with recurrent flares during pregnancy and lactation can be treated with low doseglucocorticoids for prophylaxis. Allopurinol is a category $\mathrm{C}$ teratogen since it inhibits purine synthesis and thereby may impair fetal growth and development; ${ }^{50}$ its use is discouraged during pregnancy. On the contrary, allopurinol can be continued in lactating females. ${ }^{51}$ However, since it can be excreted in breast milk, breastfed infants of lactating females who require treatment with allopurinol should be closely monitored for adverse processes, including hypersensitivity reactions and cytopenias. Notably, the full range of effects of allopurinol on infants remains unclear. 
Table I Summarization of Review Highlights and Opportunities for Future Research in Female Gout

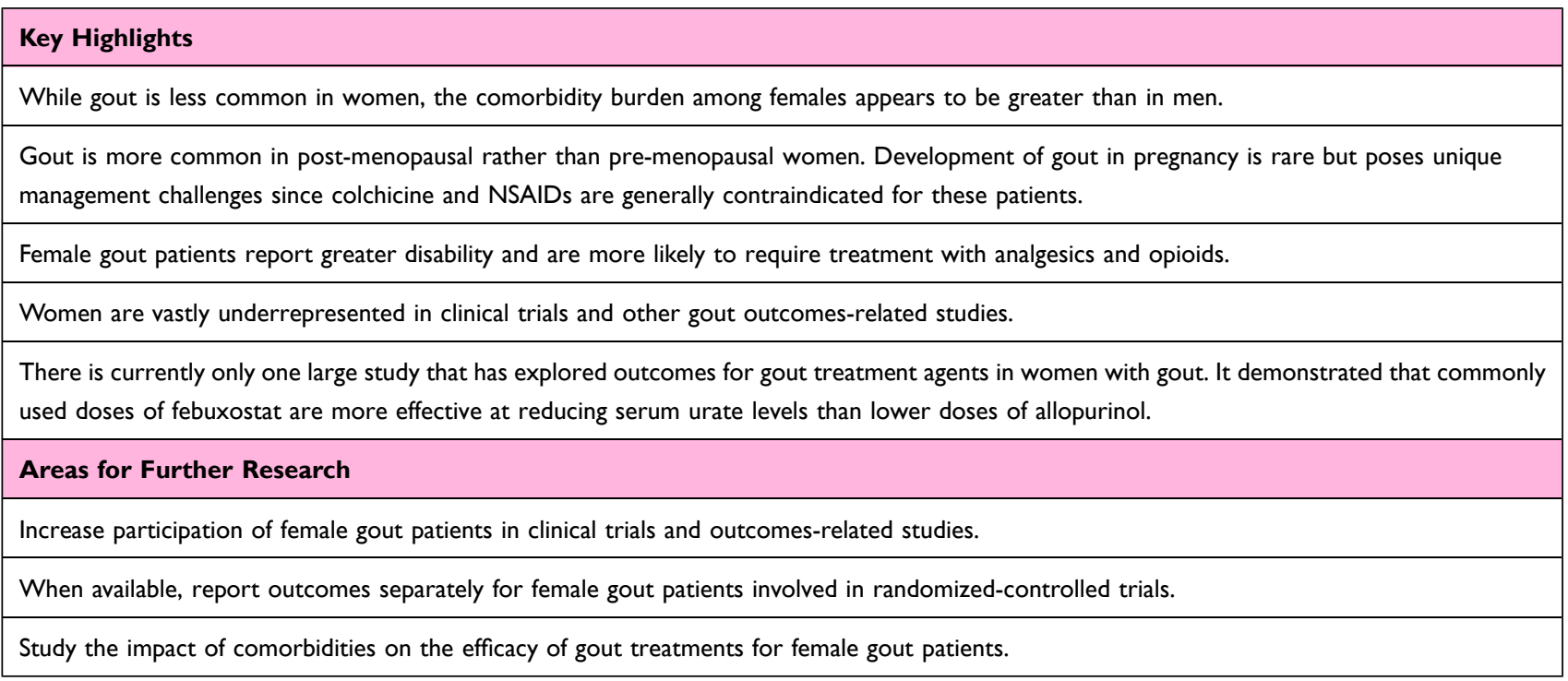

\section{Dietary Recommendations}

The risk factor profile for the development of gout in both males and females is in part driven by beef, seafood, and alcohol consumption. ${ }^{7}$ While only less than $20 \%$ of female gout patients consume beer, wine, or hard liquor, greater than $75 \%$ consume beef and dairy products, both of which have a high purine content. ${ }^{7}$ Thus, minimizing consumption of these foods could serve as a nonpharmacologic intervention that would help female gout patients achieve goal serum urate levels and reduce the frequency of flares.

\section{Conclusion}

The increased prevalence of gout in males explains the paucity of appropriately well-powered, randomizedcontrolled trials investigating the efficacy of the above therapies in female gout patients. This poses major challenges for the management of female gout patients since they carry a greater burden of cardiovascular and renal morbidity, which is known to modulate the pathophysiology of gout; as such, conclusions regarding the efficacy of treatments for females cannot be extrapolated from investigative studies that are predominantly male. Data is most abundant for xanthine oxidase inhibitor use in female patients and suggests that commonly prescribed doses of febuxostat appear to be more effective than lower doses of allopurinol, with no clear differences in cardiovascular morbidity or mortality among women treated with these two medications. Aside from this, there is only minimal data, in most cases limited to case reports, case series, or small pilot studies, which demonstrate the responsiveness of small numbers of female gout patients to uricosuric agents and uricases for chronic management and NSAIDs, colchicine, systemic steroids, and anakinra for treatment of acute flares. Lastly, flares during pregnancy and lactation are rare occurrences but pose unique challenges; in these cases, glucocorticoids are favored over other gout pharmacotherapies for safety reasons. Clinical trials and outcomes studies in gout should attempt to include more female patients and report outcomes separately for female study participants. Table 1 summarizes key highlights from this review and outlines areas in need of further research.

\section{Disclosure}

Dr Angelo L Gaffo reports personal fees and royalties from UptoDate, outside the submitted work. The authors report no other conflicts of interest in this work. 


\section{References}

1. Singh JA, Gaffo A. Gout epidemiology and comorbidities. Semin Arthritis Rheum. 2020;50(3s):S11-s16. doi:10.1016/j.semarthrit.2020.04.008

2. McCarty DJ, Hollander JL. Identification of urate crystals in gouty synovial fluid. Ann Intern Med. 1961;54:452-460.

3. Dalbeth N, Bardin T, Doherty M, et al. Discordant American College of Physicians and international Rheumatology guidelines for gout management: consensus statement of the Gout, Hyperuricemia and Crystal-Associated Disease Network (G-CAN). Nat Rev Rheumatol. 2017;13(9):561568. doi:10.1038/nrrheum.2017.126

4. FitzGerald JD, Dalbeth N, Mikuls T, et al. 2020 American College of Rheumatology Guideline for the Management of Gout. Arthritis Rheumatol. 2020;72(6):879-895. doi:10.1002/art.41247

5. Kuo C-F, Grainge MJ, Mallen C, et al. Rising burden of gout in the UK but continuing suboptimal management: a nationwide population study. Ann Rheum Dis. 2015;74(4):661-667. doi:10.1136/annrheumdis-2013-204463

6. Te Kampe R, Janssen M, van Durme C, et al. Sex differences in the clinical profile among patients with gout: cross-sectional Analyses of an Observational Study. J Rheumatol. 2021;48(2):286-292. doi:10.3899/jrheum.200113

7. Harrold LR, Etzel CJ, Gibofsky A, et al. Sex differences in gout characteristics: tailoring care for women and men. BMC Musculoskelet Disord. 2017;18(1):108. doi:10.1186/s12891-017-1465-9

8. Hak AE, Curhan GC, Grodstein F, et al. Menopause, postmenopausal hormone use and risk of incident gout. Ann Rheum Dis. 2010;69(7):13051309. doi:10.1136/ard.2009.109884

9. Roman Y, Tiirikainen M, Prom-Wormley E. The prevalence of the gout-associated polymorphism rs $2231142 \mathrm{G}>\mathrm{T}$ in $\mathrm{ABCG} 2$ in a pregnant female Filipino cohort. Clin Rheumatol. 2020;39(8):2387-2392. doi:10.1007/s10067-020-04994-9

10. Cea Soriano L, Rothenbacher D, Choi HK, et al. Contemporary epidemiology of gout in the UK general population. Arthritis Res Ther. 2011;13(2): R39. doi:10.1186/ar3272

11. Narang RK, Topless R, Cadzow M, et al. Interactions between serum urate-associated genetic variants and sex on gout risk: analysis of the UK Biobank. Arthritis Res Ther. 2019;21(1):13. doi:10.1186/s13075-018-1787-5

12. Yoo H-G, Lee S-I, Chae H-J, et al. Prevalence of insulin resistance and metabolic syndrome in patients with gouty arthritis. Rheumatol Int. 2011;31 (4):485-491. doi:10.1007/s00296-009-1304-x

13. Sumpter N, Cadzow M, So A, Reynolds R, Merriman T. Analysis of common gout comorbidities in the UK Biobank cohort reveals sex-specific effects and genetic differentiation. Arthritis Rheumatol. 2020;72:45.

14. Pillinger MH, Mandell BF. Therapeutic approaches in the treatment of gout. Semin Arthritis Rheum. 2020;50(3s):S24-s30. doi:10.1016/j. semarthrit.2020.04.010

15. Richette P, Doherty M, Pascual E, et al. 2016 updated EULAR evidence-based recommendations for the management of gout. Ann Rheum Dis. 2017;76(1):29-42. doi:10.1136/annrheumdis-2016-209707

16. Perez-Ruiz F, Dalbeth N. Combination urate-lowering therapy in the treatment of gout: what is the evidence? Semin Arthritis Rheum. 2019;48 (4):658-668. doi:10.1016/j.semarthrit.2018.06.004

17. Becker MA, Schumacher HR, Wortmann RL, et al. Febuxostat compared with allopurinol in patients with hyperuricemia and gout. $N$ Engl $J$ Med. 2005;353(23):2450-2461. doi:10.1056/NEJMoa050373

18. Schumacher HR Jr, Becker MA, Wortmann RL, et al. Effects of febuxostat versus allopurinol and placebo in reducing serum urate in subjects with hyperuricemia and gout: a 28-week, Phase III, randomized, double-blind, parallel-group trial. Arthritis Rheum. 2008;59(11):1540-1548. doi:10.1002/art.24209

19. Becker MA, Schumacher HR, Espinoza LR, et al. The urate-lowering efficacy and safety of febuxostat in the treatment of the hyperuricemia of gout: the CONFIRMS trial. Arthritis Res Ther. 2010;12(2):R63. doi:10.1186/ar2978

20. Chohan S, Becker MA, MacDonald PA, et al. Women with gout: efficacy and safety of urate-lowering with febuxostat and allopurinol. Arthritis Care Res (Hoboken). 2012;64(2):256-261. doi:10.1002/acr.20680

21. Khosravan R, Kukulka MJ, Wu J-T, et al. The effect of age and gender on pharmacokinetics, pharmacodynamics, and safety of febuxostat, a novel nonpurine selective inhibitor of xanthine oxidase. J Clin Pharmacol. 2008;48(9):1014-1024. doi:10.1177/0091270008322035

22. Mizuno T, Hayashi T, Hikosaka S, et al. Efficacy and safety of febuxostat in elderly female patients. Clin Interv Aging. 2014;9:1489-1493. doi:10.2147/CIA.S70855

23. Foody J, Turpin RS, Tidwell BA, et al. Major cardiovascular events in patients with gout and associated cardiovascular disease or heart failure and chronic kidney disease initiating a xanthine oxidase inhibitor. Am Health Drug Benefits. 2017;10(8):393-401.

24. White WB, Saag KG, Becker MA, et al. Cardiovascular safety of febuxostat or allopurinol in patients with gout. N Engl J Med. 2018;378 (13):1200-1210. doi:10.1056/NEJMoa1710895

25. Zhang M, Solomon DH, Desai RJ, et al. Assessment of cardiovascular risk in older patients with gout initiating febuxostat versus allopurinol: population-Based Cohort Study. Circulation. 2018;138(11):1116-1126. doi:10.1161/CIRCULATIONAHA.118.033992

26. Mackenzie IS, Ford I, Nuki G, et al. Long-term cardiovascular safety of febuxostat compared with allopurinol in patients with gout (FAST): a multicentre, prospective, randomised, open-label, non-inferiority trial. Lancet. 2020;396(10264):1745-1757. doi:10.1016/S0140-6736(20)32234-0

27. Pui K, Gow PJ, Dalbeth N. Efficacy and tolerability of probenecid as urate-lowering therapy in gout; clinical experience in high-prevalence population. J Rheumatol. 2013;40(6):872-876. doi:10.3899/jrheum.121301

28. Richette P, Bardin T. Successful treatment with rasburicase of a tophaceous gout in a patient allergic to allopurinol. Nat Clin Pract Rheumatol. 2006;2(6):338-342; quiz 343. doi:10.1038/ncprheum0214

29. Vogt B. Urate oxidase (rasburicase) for treatment of severe tophaceous gout. Nephrol Dial Transplant. 2005;20(2):431-433. doi:10.1093/ndt/ gfh629

30. Wipfler-Freibmuth E, Dejaco C, Duftner C, et al. Urate oxidase (rasburicase) for treatment of severe acute gout: a case report. Clin Exp Rheumatol. 2009;27(4):658-660.

31. Sundy JS, Baraf HS, Yood RA, et al. Efficacy and tolerability of pegloticase for the treatment of chronic gout in patients refractory to conventional treatment: two randomized controlled trials. JAMA. 2011;306(7):711-720. 
32. Sundy JS, Becker MA, Baraf HSB, et al. Reduction of plasma urate levels following treatment with multiple doses of pegloticase (polyethylene glycol-conjugated uricase) in patients with treatment-failure gout: results of a Phase II randomized study. Arthritis Rheum. $2008 ; 58(9): 2882-2891$. doi:10.1002/art.23810

33. Araujo EG, Bayat S, Petsch C, et al. Tophus resolution with pegloticase: a prospective dual-energy CT study. RMD Open. 2015;1(1):e00075. doi:10.1136/rmdopen-2015-000075

34. Rubin BR, Burton R, Navarra S, et al. Efficacy and safety profile of treatment with etoricoxib 120 mg once daily compared with indomethacin 50 mg three times daily in acute gout: a randomized controlled trial. Arthritis Rheum. 2004;50(2):598-606. doi:10.1002/art.20007

35. Navarra S, Rubin BR, Yu Q, et al. Association of baseline disease and patient characteristics with response to etoricoxib and indomethacin for acute gout. Curr Med Res Opin. 2007;23(7):1685-1691. doi:10.1185/030079907X210750

36. Terkeltaub RA, Furst DE, Bennett K, et al. High versus low dosing of oral colchicine for early acute gout flare: twenty-four-hour outcome of the first multicenter, randomized, double-blind, placebo-controlled, parallel-group, dose-comparison colchicine study. Arthritis Rheum. 2010;62 (4):1060-1068. doi:10.1002/art.27327

37. Fiolet ATL, Opstal TSJ, Mosterd A, et al. Efficacy and safety of low-dose colchicine in patients with coronary disease: a systematic review and meta-analysis of randomized trials. Eur Heart J. 2021;42(28):2765-2775. doi:10.1093/eurheartj/ehab115

38. Nidorf SM, Fiolet ATL, Mosterd A, et al. Colchicine in patients with chronic coronary disease. $N$ Engl J Med. $2020 ; 383(19): 1838-1847$. doi:10.1056/NEJMoa2021372

39. Groff GD, Franck WA, Raddatz DA. Systemic steroid therapy for acute gout: a clinical trial and review of the literature. Semin Arthritis Rheum. 1990;19(6):329-336. doi:10.1016/0049-0172(90)90070-V

40. So A, De Smedt T, Revaz S, et al. A pilot study of IL-1 inhibition by anakinra in acute gout. Arthritis Res Ther. 2007;9(2):R28. doi:10.1186/ar2143

41. Chen K, Fields T, Mancuso CA, et al. Anakinra's efficacy is variable in refractory gout: report of ten cases. Semin Arthritis Rheum. 2010;40 (3):210-214. doi:10.1016/j.semarthrit.2010.03.001

42. Janssen CA, Oude Voshaar MAH, Vonkeman HE, et al. Anakinra for the treatment of acute gout flares: a randomized, double-blind, placebocontrolled, active-comparator, non-inferiority trial. Rheumatology (Oxford. 2019;58(8):1344-1352. doi:10.1093/rheumatology/key402

43. Thueringer JT, Doll NK, Gertner E. Anakinra for the treatment of acute severe gout in critically ill patients. Semin Arthritis Rheum. 2015;45(1):8185. doi:10.1016/j.semarthrit.2015.02.006

44. Liew JW, Gardner GC. Use of anakinra in hospitalized patients with crystal-associated arthritis. J Rheumatol. 2019;46(10):1345-1349. doi:10.3899/ jrheum. 181018

45. Lhotta K, Gehringer A, Jennings P, et al. Familial juvenile hyperuricemic nephropathy: report on a new mutation and a pregnancy. Clin Nephrol. 2009;71(1):80-83. doi:10.5414/CNP71080

46. Simmonds HA, Cameron JS, Goldsmith DJ, et al. Familial juvenile hyperuricaemic nephropathy is not such a rare genetic metabolic purine disease in Britain. Nucleosides Nucleotides Nucleic Acids. 2006;25(9-11):1071-1075. doi:10.1080/15257770600891028

47. van Veen TR, Haeri S. Gout in pregnancy: a case report and review of the literature. Gynecol Obstet Invest. 2015;79(4):217-221. doi:10.1159/ 000369999

48. Ben-Chetrit E, Scherrmann JM, Levy M. Colchicine in breast milk of patients with familial Mediterranean fever. Arthritis Rheum. 1996;39 (7):1213-1217. doi:10.1002/art.1780390721

49. Adler Y, Charron P, Imazio M, et al. 2015 ESC guidelines for the diagnosis and management of pericardial diseases: the task force for the diagnosis and management of pericardial diseases of the European Society of Cardiology (ESC) Endorsed by: the European Association for Cardio-Thoracic Surgery (EACTS). Eur Heart J. 2015;36(42):2921-2964. doi:10.1093/eurheartj/ehv318

50. Fazal MW, Doogue MP, Leong RW, et al. Allopurinol use in pregnancy in three women with inflammatory bowel disease: safety and outcomes: a case series. BMC Gastroenterol. 2013;13:172. doi:10.1186/1471-230X-13-172

51. Allopurinol. Drugs and Lactation Database (Lactmed). Bethesda (MD): National Library of Medicine (US); 2006.

Journal of Inflammation Research

\section{Publish your work in this journal}

The Journal of Inflammation Research is an international, peer-reviewed open-access journal that welcomes laboratory and clinical findings on the molecular basis, cell biology and pharmacology of inflammation including original research, reviews, symposium reports, hypothesis formation and commentaries on: acute/chronic inflammation; mediators of inflammation; cellular processes; molecular mechanisms; pharmacology and novel anti-inflammatory drugs; clinical conditions involving inflammation. The manuscript management system is completely online and includes a very quick and fair peer-review system. Visit http://www.dovepress.com/testimonials.php to read real quotes from published authors.

Submit your manuscript here: https://www.dovepress.com/journal-of-inflammation-research-journal 